Article

\title{
A Model of Interaction between Nicotinamide Adenine Dinucleotide Phosphate (NADPH) Oxidase and Apocynin Analogues by Docking Method
}

Jie Jiang ${ }^{1}$, Hongjun Kang ${ }^{1, \dagger}$, Xiaoliang Song ${ }^{1, \dagger}$, Sichao Huang ${ }^{1}$, Sha $\mathrm{Li}^{1, *}$ and Jun $\mathrm{Xu}^{2, *}$

1 College of Pharmacy, Jinan University, Guangzhou 510632, China;

E-Mails: jiejiang2008@gmail.com (J.J.); hongjunkang@126.com (H.K.);

Song1xiaoliang@sina.com (X.S.); sichaohuang.cn@gmail.com (S.H.)

2 College of Medicine, Jinan University, Guangzhou 510632, China

$\dagger$ These authors contributed equally to this work.

* Authors to whom correspondence should be addressed; E-Mails: tlisha@jnu.edu.cn (S.L.); goldstar_8209@163.com (J.X.); Tel.: +86-020-8522-3784 (S.L.); +86-020-8522-3704 (J.X.).

Received: 14 November 2012; in revised form: 11 December 2012 / Accepted: 24 December 2012 / Published: 4 January 2013

\begin{abstract}
Some apocynin analogues have exhibited outstanding inhibition to NADPH oxidase. In this study, the key interactions between apocynin analogues and NADPH oxidase were analyzed by the docking method. The potential active site was first identified by the SiteID program combining with the key residue CYS378. Afterwards, the compounds in the training set were docked into NADPH oxidase $(1 \mathrm{~K} 4 \mathrm{U})$ under specific docking constraints to discuss the key interactions between ligands and the receptor. These key interactions were then validated by the consistence between the docking result and the experimental result of the test set. The result reveals that the Pi interaction between apocynin analogues and NADPH oxidase has a direct contribution to inhibition activities, except for H-bond formation and docking score. The key interactions might be valuable to discover and screen apocynin analogues as potent inhibitors of NADPH oxidase.
\end{abstract}

Keywords: NADPH oxidase; apocynin analogues; inhibitor; docking 


\section{Introduction}

NADPH oxidase, initially characterized in neutrophils, is a multimeric enzyme consisting of gp91phox (renamed Nox2), p22phox, 3 cytosolic subunits (p47phox, p67phox and p40phox) and the small G-proteins (Rac1/2 and Rap1A) [1,2]. Mutations studies on NADPH oxidase suggest that p47phox requires at least three serines for oxidase activation as follows: a serine at position 379, a phosphorylated serine at position 359 or 370 and a phosphorylated serine at position 303 or 304 [3]. The enzyme plays a key role in immunological host defense for its responsibility to produce reactive oxygen species (ROS). To atherosclerosis, the endothelial NADPH oxidase plays also an important role. By generating superoxidanions, which immediately react with NO to the highly reactive peroxynitrite, the NADPH oxidase is the main source for endothelial dysfunction (an early key event in atherosclerosis) [4-6]. Meanwhile, inhibition of this enzyme represents an attractive therapeutic approach for the treatment of cardiovascular disease, acute lung injury and so on $[7,8]$.

Apocynin (4-hydroxy-3-methoxyacetophenone), originally extracted from the roots of Picrorhiza kurroa, has been extensively used for the treatment of diseases associated with chronic inflammation, including jaundice, asthma and cardiovascular diseases [9,10]. Current studies have revealed that apocynin is an efficient non-toxic inhibitor of NADPH oxidase [11]. Therefore, a series of apocynin analogues were synthesized by traditional methods, and their inhibition activities were also tested in vitro $[12,13]$. These works about apocynin analogues have uncovered some potential NADPH oxidase inhibitors better than apocynin and indicated that apocynin would be a valuable lead compound. Some structure-function analyses reveal that the mechanism of apocynin inhibition to NADPH oxidase is a result of peroxidase metabolism, yielding reactive quinones that bind to Cys residues in $\mathrm{p} 47 \mathrm{phox}$ and impeding the migration of the cytosolic component of p47phox to the membrane [14-16]. Moreover, mutations of Cys378, which is treated as a conserved residue [17], or one of the other three p47phox cysteines have direct effects in NADPH oxidase activation [18]. The previous studies provide some valuable information that should be constructive to discover and design novel inhibitors of NADPH oxidase. To accelerate the development of apocynin analogues as NADPH oxidase inhibitors, the key interactions between inhibitors and receptor should be analyzed based on the compounds reported.

Compared with traditional methods to explore the interaction between ligands and receptors, the docking method can effectively shorten the cycle time of research and reduce costs. For these reasons, the docking method has been widely used to identify the key interactions between ligands and their receptors [19-22]. The known important interactions would be useful to discover more candidates and guide the synthesis of novel targeted compounds. In this study, the docking method was utilized to explore and validate the key interactions between apocynin analogues and NADPH oxidase combining with our previous experimental result.

\section{Computational Methods}

\subsection{Database and Software}

The structure and inhibitory activities (IC50) of 12 compounds, which served as the training set, were collected from the literature [13]. The test set was composed $\mathrm{f} 11$ apocynin analogues synthesized by our group [12]. The 3D structure of NADPH oxidase $(1 \mathrm{~K} 4 \mathrm{U})$ was derived from the RCSB protein 
data bank and consisted of the $C$-terminal SH3 domain of p67phox complexed with the $C$-terminal tail region of p47phox [23]. All the compounds collected in this study would be docked into the 3D structure of $1 \mathrm{~K} 4 \mathrm{U}$ by the GOLD (Genetic Optimisation for Ligand Docking) program (CCDC Co., Ltd., Cambridge, UK), which is an effective docking program for docking ligands into protein binding sites based on genetic algorithms [24,25].

\subsection{Preparation of Inhibitors}

The structures of ligands have been found to be one of the important determinants for a successful docking. Thus, making pretreatments of inhibitors, such as energy minimization and analysis of the structures, is necessary. First of all, all the 23 ligands' structures were drawn by sybyl7.0 (Tripos Co., Ltd., St. Louis, MO, USA) and make sure the atoms' type and chirality is correct. Moreover, the structures' energy was optimized by the Powell method, and the charges were calculated by the MMFF94 method in the Tripos force field [26-28]. The compounds in the training set were divided into two categories. Relative good inhibitors consisted of the top six inhibition activity compounds, and the other six compounds were treated as relative weak inhibitors.

\subsection{Identification of the Potential Active Site}

The preparation of the receptor and identification of the active site of NADPH oxidase also have a direct contribution to an ideal docking result. Therefore, the 3D structure of NADPH oxidase should be protonated and the water removed primarily. The loop definition, minimization and calculation of charge were then carried out in the Tripos field. After preparation of the NADPH oxidase 3D structure, the SiteID program of Syby17.0 was applied to search the potential binding sites on the NADPH oxidase by a flood-fill solvation technique [29]. It has been reported that CYS378 was a key residue in the binding site $[14,15,17,18]$, which would be useful to identify the potential active site according to the results of searching the potential binding site.

\subsection{Construction and Validation of the Docking Condition}

In the premise of the identification of the potential active site with key residue CYS378, the compounds in training set were docked into NADPH oxidase by the GOLD program. In general, an ideal docking result has a close relation with suitable docking constraints, such as the constrained region for the hydrophobic group, ligand flexibility, receptor flexibility, soft potential, H-bond and the docking pose. Hydrogen bond interactions are considered to be key interactions between the N-SH3 domain of p47phox and PRR of p22phox [30,31]. Similar interactions between inhibitors and p47phox may disrupt the association of $\mathrm{p} 47 \mathrm{phox}-\mathrm{p} 21$ phox, which has a direct contribution to NADPH oxidase activity. Therefore, the carbonyl and amino group of the key residue CYS378 of p47phox were treated as a possible $\mathrm{H}$-bone former in the docking process. The other residues in the potential active site were set to be rigid, but compounds in our ligand database were regarded to be flexible, which served as other docking constraints. Compounds with strong inhibition activities to NADPH oxidase usually have stronger interaction with NADPH oxidase compared with the ones with weak inhibition activities. And, docking score is widely used to quantify the interaction between ligand and receptor: a 
higher score reflects a stronger interaction. Therefore, the docking score should exhibit favorable consistence with inhibition activities of NADPH oxidase. GoldScore has been confirmed to be a high performance docking score [32], so the score function was taken to evaluate the interaction between NADPH oxidase and its inhibitors, as well as inhibition activities in this study.

All the compounds in the training set were docked into the potential active site of NADPH oxidase, identified by SiteID program, under the docking constraints discussed above. After the calculation of the docking score, the docking conformation with the best score was taken as the targeted conformation for each compound in the training set, as well as the test set. At the same time, these compounds were also divided into two groups according to the value of the docking score. The top six docking score compounds were taken as predicted good inhibitors, and the other six compounds were treated as predicted weak inhibitors. As the construction of docking condition was utilized to discover the potent inhibitors of NADPH oxidase, the relative good inhibitors classified by $\mathrm{IC}_{50}$ were compared with predicted good inhibitors identified by the docking score to figure out how many predicted good inhibitors would be real good inhibitors. More of these kinds of compounds might show the better prediction ability of this docking method for discovering good inhibitors. For further analysis of the key interaction between NADPH oxidase and its inhibitors, the possible interactions between targeted docking conformation and receptor were discussed for each compound in the training set. Based on the hypothesis of key interaction deduced from the training set, the compounds in the test set were docked into NADPH oxidase to validate the hypothesis. In the validation process, the targeted conformations of compounds in the test set were used to analyze the potential interactions with the receptor. Among these interactions, the correlation between hypothesized key interactions and inhibition activities tested in vitro was further explored.

Inhibition activities to NADPH in vitro of the compounds in the test set were evaluated by MTT assay in our previous studies [11]. RAW 264.7 cells were seeded into a 96-well microplate at a density of $2.0 \times 10^{4}$ cells/well. The cells were cultured and treated with various doses of compounds $(0.1,1$, $10,100 \mu \mathrm{M})$ for $1 \mathrm{~h}$ prior to the addition of LPS. After $24 \mathrm{~h}$ of incubation, MTT was then added $(0.5 \mathrm{mg} / \mathrm{mL})$ for $4 \mathrm{~h}$, the medium was removed and the formazan crystals were dissolved in DMSO and isopropanol (1:1). Optical density of the solution in each well was measured at $540 \mathrm{~nm}$. The cell viability rate was calculated as the percentage of MTT absorbance

\section{Results and Discussion}

\subsection{Training Set and Test Set}

Figure 1 illustrates structures and inhibition activities $\left(\mathrm{IC}_{50}\right)$ of the compounds in the training set collected from literature. Analysis of the structures of compounds in the training set reveal that they are all apocynin derivatives and have similar structure fragments. The similarity of structures usually results in a similar interaction or binding style between ligand and receptor. Thus, it is reasonable to believe that these compounds in the training set should bind to the same active site as apocynin. According to the inhibition activities of these compounds in the training set, apocynin dimer, homovanillin alcohol, tyrosol, ferulic acid, hydroxytyrosol and caffeic acid are treated as relative good inhibitors, while others are relative weak inhibitors. 
Figure 1. Structures and inhibition activities (IC50: $\mu \mathrm{M}$ ) of apocynin analogues as the training set.
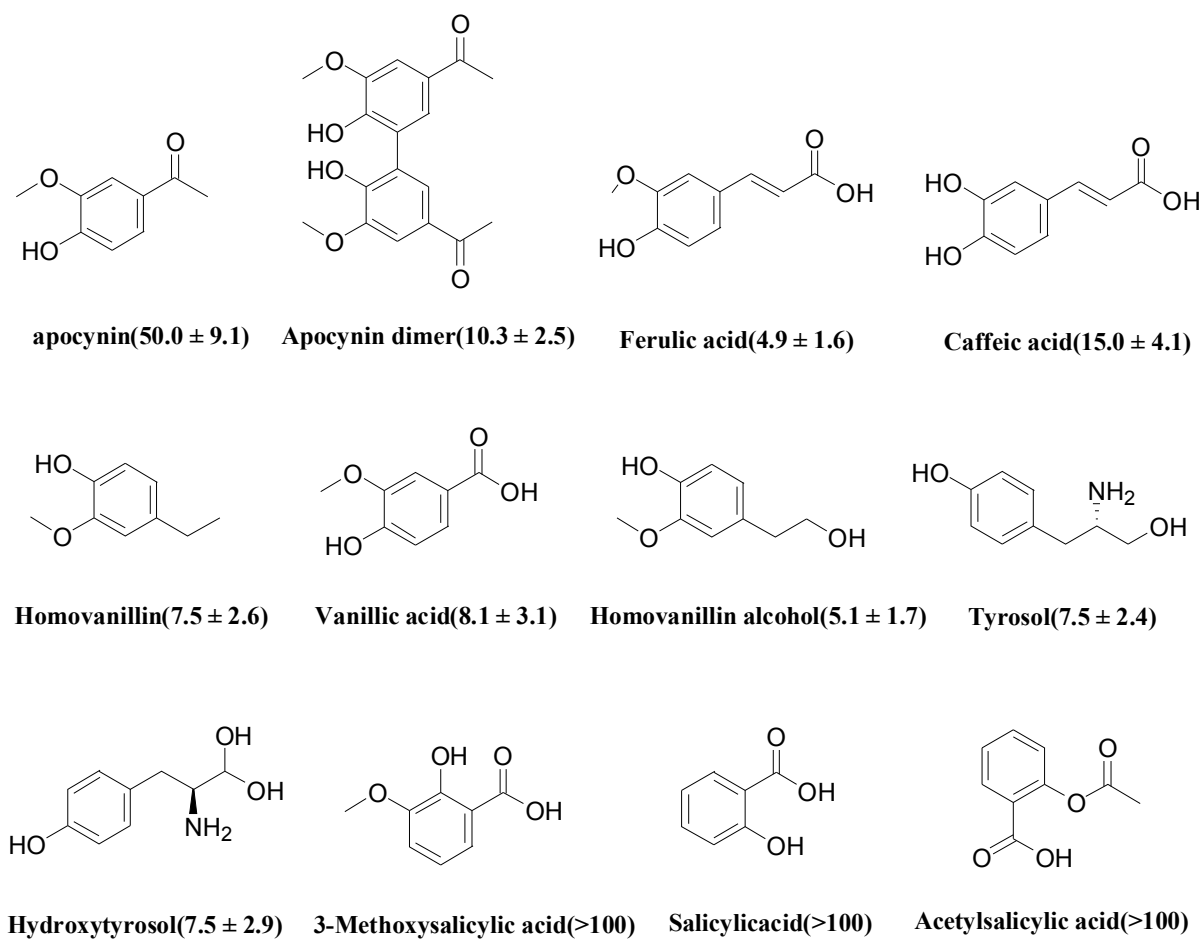

For the potent inhibition activities of some apocynin derivatives, some new analogues were synthesized in our previous work to discover a stronger NADPH oxidase inhibitor (Figure 2). Inhibition activities of these new derivatives to NADPH were also tested in vitro, and the result would be valuable to validate the hypothesis concluded from the docking result. In this study, these compounds would be served as a test set, and the consistence between docking result and experimental result was used to validate the interaction model.

Figure 2. Apocynin analogues synthesized in our group as the test set.
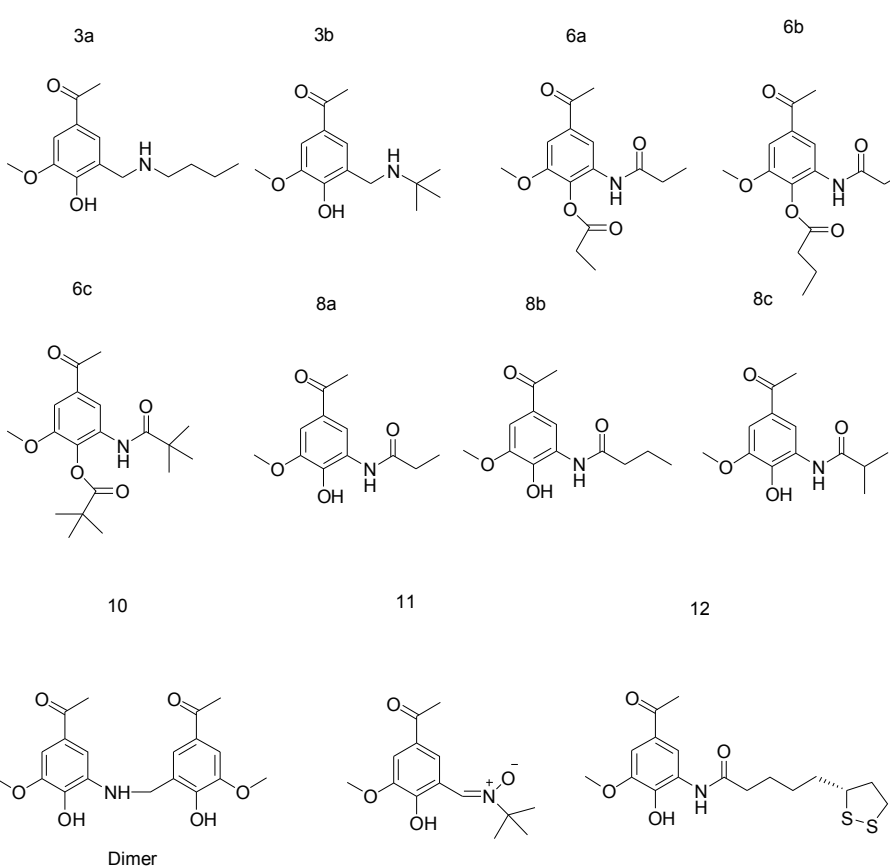


\subsection{Identification of the Potential Active Site}

The potential active site is the premise of the docking study about the interaction between NADPH oxidase and its inhibitors. Although some works have been done on the interaction between NADPH and apocynin, the potential active site is not clear, except for some key residues. From the key residues reported, CYS378 is accepted as the key residue to have a close relationship with inhibition activities of inhibitors $[14,15,17,18]$. Generally, the active site is the pocket formed by a series of conservative amino acid in or on the receptor. Therefore, the SiteID program was used to seek all the possible pockets in or on 1K4U using a flood-fill salvation technique. The Table 1 lists seven pockets identified by SiteID and residues composing the pockets. Considering that CYS378 has been reported as the key residue, the No. 6 pocket containing CYS378 is chosen as the potential active site in this study.

Table 1. The pockets in or on $1 \mathrm{~K} 4 \mathrm{U}$ identified by SiteID.

\begin{tabular}{cl}
\hline Pocket & \multicolumn{1}{c}{ Residues } \\
\hline 1 & GLN461, VAL462, VAL486, LYS489, GLU492, TRP494, LEU495, LYS508, ASP531, SER460, THR516 \\
2 & GLN461, VAL462, GLU463, ILE483, ILE484, LEU485, LYS458, SER514, SER460, ALA515 \\
3 & PRO363, ALA364, PRO366, PRO367, ARG368, PRO369, ASN491, GLU492, GLU493, TRP494, LYS508 \\
4 & GLN362, PRO363, ALA364, VAL365, PRO366, ARG368, SER467, TYR468, GLU469, GLN472, ASP475, TRP494, PHE510 \\
5 & LYS385, ALA470, GLN472, PRO473, GLU474, ASP475, LEU476, GLU477, PHE478, GLN479, ASP482, ILE484, GLY497, \\
& SER499, LYS500, LYS502, VAL503, GLY504 \\
7 & ARG377, CYS378, SER379, LEU487, SER488, LYS489, GLU496 \\
\hline
\end{tabular}

\subsection{Molecular Docking of the Training Sets}

After the identification of the potential active site, the compounds in the training set were docked into $1 \mathrm{~K} 4 \mathrm{U}$ by GOLD, as described in the methods section. In the process, CYS378 treated as H-bond former was the major docking constraint. Docking conformation with the best score was picked out as the preference conformation for each compound. These preference conformations will be taken into subsequent analysis of the key interaction, and their scores are listed in Table 2. In Table 2, relative good inhibitors classified by $\mathrm{IC}_{50}$ are emphasized by italics, while the good inhibitors being also predicted as good inhibitors are emphasized in italic and bold type. There are four relative good inhibitors in six predicted good inhibitors, and the remaining compounds also have, to some extent, obvious inhibition activities to NADPH oxidase. The result suggests that the docking score can distinguish the good and weak inhibitors under the specific docking constraints in this study, which also support the importance of H-bond formation between apocynin analogues and the CYS378 of NADPH oxidase. Based on the result, the key interactions between these compounds in the training set and $1 \mathrm{~K} 4 \mathrm{U}$ were further analyzed, especially for the four good inhibitors supported by both $\mathrm{IC}_{50}$ and the docking score. Figure 3 illustrated the potential interactions between predicted good inhibitors and NADPH oxidase. In the figure, H-bonds between ligand and receptor are marked by a dotted arrow, while H-bond acceptors of ligand are colored by blue and green color, indicating the H-bond donor. Beside the H-bonds, Pi interaction between the ligand and the receptor are emphasized by an orange line. The result demonstrates that the interesting Pi interactions between these four compounds and 
LYS383 of 1K4U are all discovered, but not observed in other predicted good compounds, such as apocynin dimer and caffeic acid. Meanwhile, the Pi interactions were also not found between the predicted weak compounds in the training set and 1K4U, except homovanillin and the SER379 of $1 \mathrm{~K} 4 \mathrm{U}$. Furthermore, homovanillin is also identified as a relative good inhibitor according to $\mathrm{IC}_{50}$. This result implies that the Pi interaction between apocynin analogues and NADPH oxidase might have a direct contribution to their inhibition activities.

Table 2. Scoring results of the molecular docking training set.

\begin{tabular}{|c|c|c|c|c|c|}
\hline & & \multirow{2}{*}{ Compound } & NADPH oxidation & \multirow{2}{*}{ GOLD Score } & \multirow{2}{*}{ Pi interaction } \\
\hline & & & $\mathrm{IC}_{50}{ }^{\mathrm{a}}(\boldsymbol{\mu m})($ Experimental $)$ & & \\
\hline \multirow{12}{*}{. } & \multirow{6}{*}{ 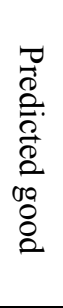 } & Apocynin dimer & $10.3 \pm 2.5$ & 45.0082 & - \\
\hline & & Homovanillin alcohol & $5.1 \pm 1.7$ & 35.1074 & LYS383 \\
\hline & & Tyrosol & $7.5 \pm 2.4$ & 32.8184 & LYS383 \\
\hline & & Ferulic acid & $4.9 \pm 1.6$ & 31.7482 & LYS383 \\
\hline & & Hydroxytyrosol & $7.5 \pm 2.9$ & 28.9217 & LYS383 \\
\hline & & Caffeic acid & $15.0 \pm 4.1$ & 27.8341 & - \\
\hline & \multirow{6}{*}{ 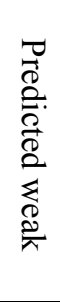 } & 3-Methoxysalicylic acid & $>100$ & 27.3678 & - \\
\hline & & Apocynin & $50.0 \pm 9.1$ & 27.0364 & - \\
\hline & & Vanillic acid & $8.1 \pm 3.1$ & 26.6326 & - \\
\hline & & Homovanillin & $7.5 \pm 2.6$ & 26.0688 & SER379 \\
\hline & & Acetylsalicylic acid & $>100$ & 25.7012 & - \\
\hline & & Salicylic acid & $>100$ & 23.2333 & - \\
\hline \multirow{11}{*}{ 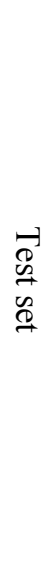 } & & 12 & - & 52.0590 & LYS383 \\
\hline & & $6 b$ & - & 42.8667 & - \\
\hline & & 10 & - & 41.1901 & LYS383 \\
\hline & & $6 c$ & - & 40.7741 & - \\
\hline & & $8 \mathrm{c}$ & - & 40.6892 & - \\
\hline & & $3 b$ & - & 38.6765 & - \\
\hline & & $8 \mathrm{a}$ & - & 38.2455 & - \\
\hline & & $8 b$ & - & 37.7346 & - \\
\hline & & 11 & - & 37.4821 & - \\
\hline & & $6 a$ & - & 36.7572 & - \\
\hline & & $3 a$ & - & 36.4118 & - \\
\hline
\end{tabular}

${ }^{\text {a }}$ Concentration $(\mu \mathrm{m})$ of inhibitors in training set required to achieve $50 \%$ inhibition of NADPH oxidase activity.

Figure 3. The key interactions between predicted good inhibitors and 1K4U.
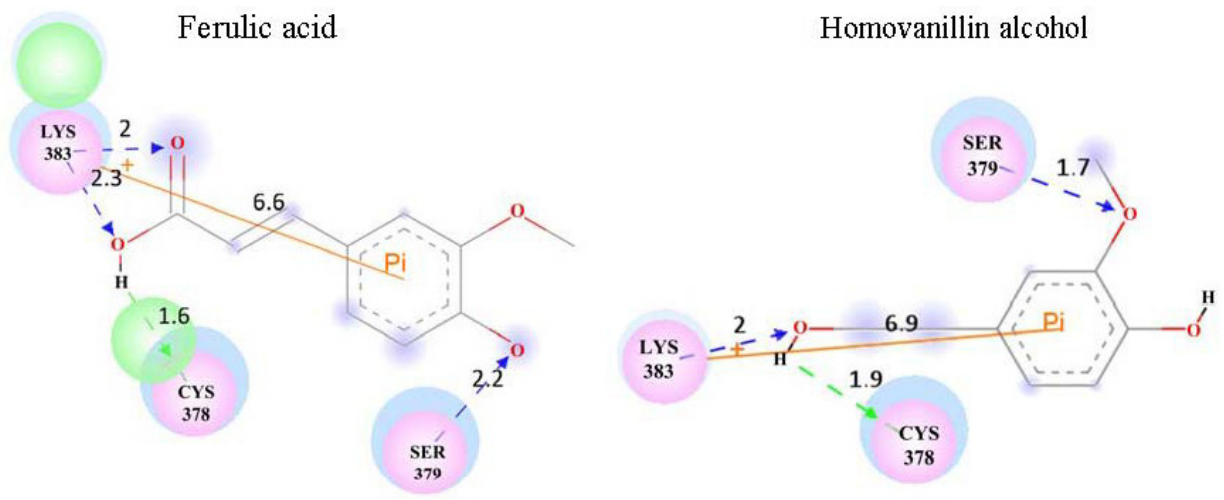
Figure 3. Cont.

Hydroxytyrosol

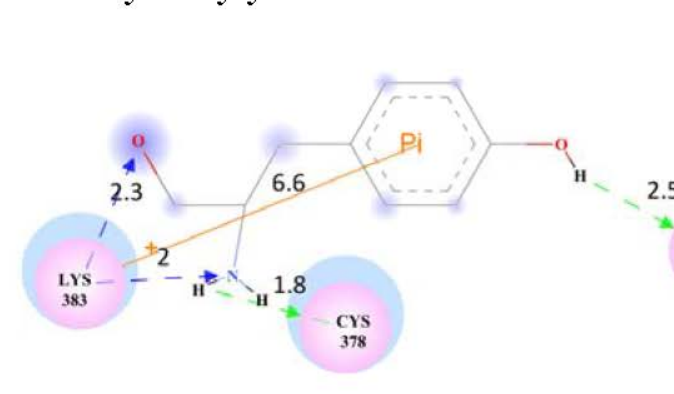

10

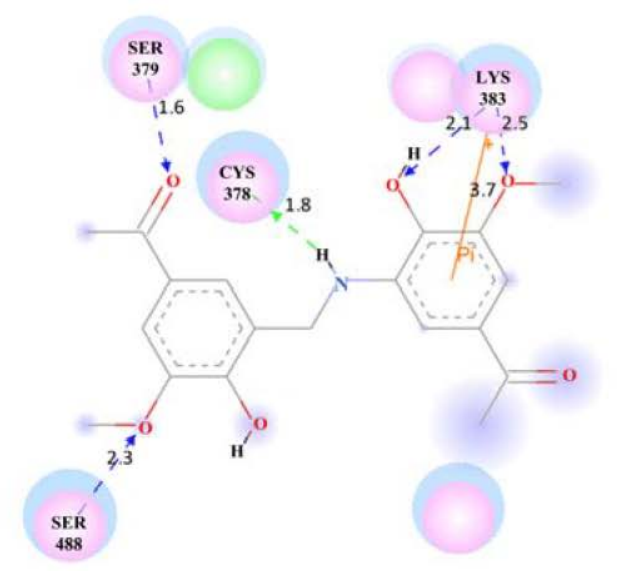

Tyrosol

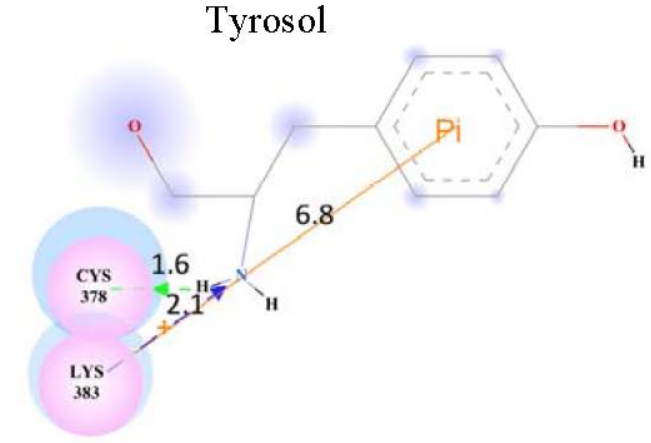

12

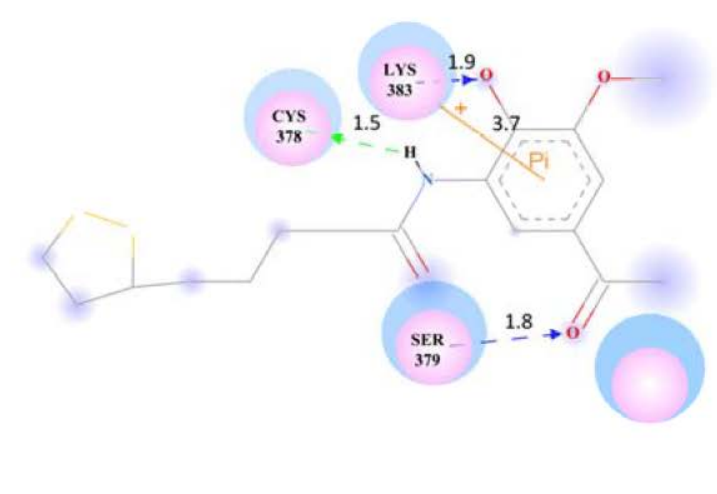

\subsection{Validation of the Hypothesis}

Based on the docking result and analysis of the interaction, a hypothesis was put forward to screen and discover apocynin analogues as potent inhibitors to NADPH oxidase. In the hypothesis, the better inhibition activity is supported by Pi interaction, as well as the better docking score and H-bond formation with CYS378. To validate the hypothesis concluded from docking study between the training set and $1 \mathrm{~K} 4 \mathrm{U}$, the compounds in the test set were docked into $1 \mathrm{~K} 4 \mathrm{U}$ by the GOLD program under the same docking constraints. The docking scores were calculated and listed in Table 2. Moreover, the interactions between these compounds and $1 \mathrm{~K} 4 \mathrm{U}$ were analyzed to find out which compounds have $\mathrm{Pi}$ interaction and H-bond formation with the receptor, except for the better docking score. The result reveals that No. 10 and 12 have a Pi interaction with LYS383 of 1K4U, just as the four compounds in the training set (Figure 3). Simultaneously, the two compounds also show a top 1 and top 3 docking score in the test set and H-bond formation with CYS378. Thus, No. 10 and 12 are chosen as potent NADPH oxidase inhibitors according to the hypothesis concluded above. It is encouraging that the predicted result is validated by our previous work about the inhibition activities of compounds in the test set (Figure 4) [12]. Inhibition to NADPH oxidase can effectively protect against LPS (lipopolysaccharide)-induced cytotoxicity in RAW 264.7 macrophage cells and promote cell viability. Therefore, better cell viability might suggest better inhibition to NADPH oxidase. As shown in Figure 4, the result reveals that No. 10 and 12 compounds have obvious better inhibition activities to NADPH oxidase in vitro compared with apocynin and other compounds in the test set. The consistency between predicted and experimental results demonstrates the hypothesis about the 
importance of Pi interaction. Meanwhile, the selection of potent inhibitors of NADPH oxidase should not only depend on H-bond formation with CYS378 and the docking score, but also the Pi interaction.

Figure 4. Protective effects against LPS (lipopolysaccharide)-induced cytotoxicity in RAW 264.7 macrophage cells [12].

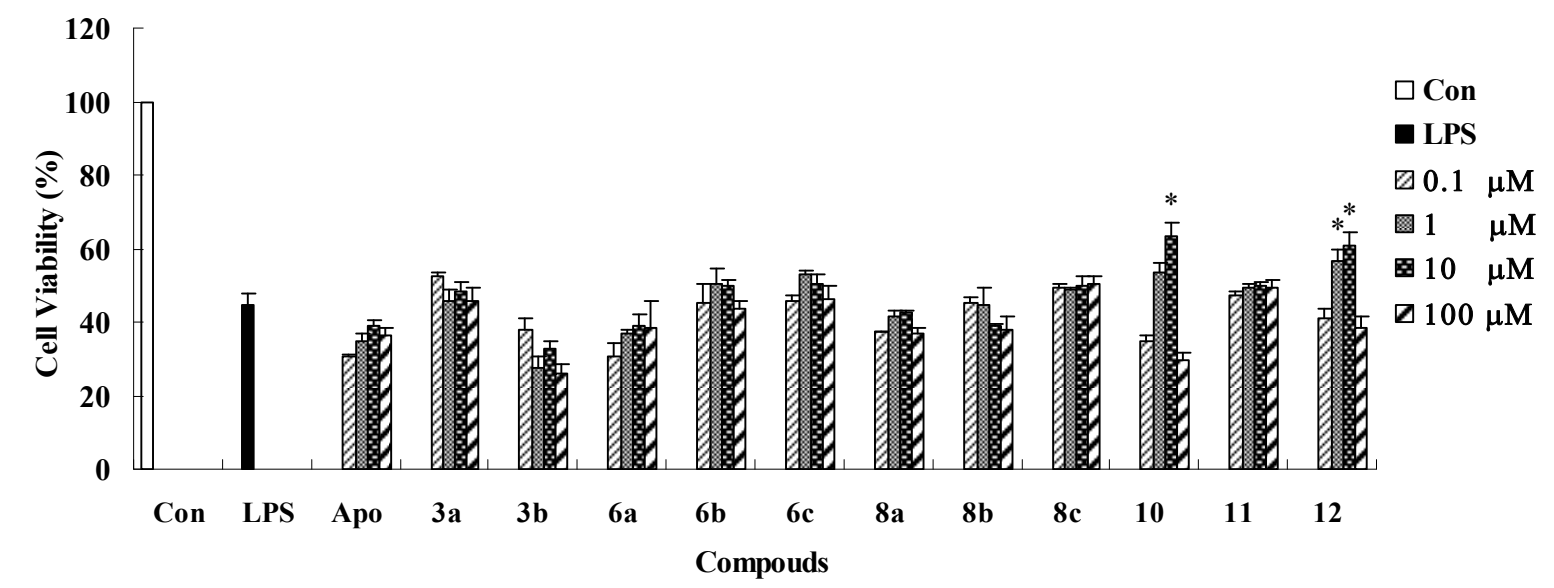

\section{Conclusions}

In this paper, a model of interaction between NADPH oxidase and apocynin analogues was constructed by the docking method. A potential active site was identified by the SiteID program and the key residue reported from previous studies. Based on the active site, the compounds in the training set were docked into NADPH oxidase $(1 \mathrm{~K} 4 \mathrm{U})$ following the specific docking constraints by GOLD. The docking result reveals that the docking score can well distinguish the good and weak inhibitors, while the further analysis of interaction between apocynin analogues in the training set and $1 \mathrm{~K} 4 \mathrm{U}$ demonstrates that $\mathrm{Pi}$ interaction might have a direct contribution to inhibition activities, except for $\mathrm{H}$-bond formation and the docking score. And, the hypothesis was supported by the consistency between the docking result of the test set and our previous experimental test in vitro. Therefore, it is reasonable to believe that the interaction model should be helpful to discover and screen apocynin analogues as potent inhibitors of NADPH oxidase.

\section{Acknowledgements}

This study was supported by grants from the China Natural Science Fund (81202461 to JX), the Funds from Department of Education of Guangdong Province (2010CXZD1012 to JJ), Guangzhou Science and Technology Funds (2011Y1-00017-10 to JJ), as well as the 211 Project of Jinan University.

\section{References}

1. De Almeida, A.C.; Cabral, M.O.; Arslanian, C.; Condino-Neto, A.; Ximenes, V.F. 4-fluoro-2-methoxyphenol, an apocynin analog with enhanced inhibitory effect on leukocyte oxidant production and phagocytosis. Eur. J. Pharmacol. 2011, 660, 445-453.

2. Kanegae, M.P.; da Fonseca, L.M.; Brunetti, I.L.; Silva, S.O.; Ximenes, V.F. The reactivity of ortho-methoxy-substituted catechol radicals with sulfhydryl groups: Contribution for the 
comprehension of the mechanism of inhibition of NADPH oxidase by apocynin. Biochem. Pharmacol. 2007, 74, 457-464.

3. Johnson, J.L.; Park, J.W.; Benna, J.E.; Faust, L.P.; Inanami, O.; Babior, B.M. Activation of p47(PHOX), a cytosolic subunit of the leukocyte NADPH oxidase. Phosphorylation of ser-359 or ser-370 precedes phosphorylation at other sites and is required for activity. J. Biol. Chem. 1998, 273, 35147-35152.

4. Steffen, Y.; Vuillaume, G.; Stolle, K.; Roewer, K.; Lietz, M.; Schueller, J.; Lebrun, S.; Wallerath, T. Cigarette smoke and LDL cooperate in reducing nitric oxide bioavailability in endothelial cells via effects on both eNOS and NADPH oxidase. Nitric Oxide 2012, 27, 176-184.

5. Cai, H.; Griendling, K.K.; Harrison, D.G. The vascular NAD(P)H oxidases as therapeutic targets in cardiovascular diseases. Trends Pharmacol. Sci. 2003, 24, 471-478.

6. Griendling, K.K.; Minieri, C.A.; Ollerenshaw, J.D.; Alexander, R.W. Angiotensin II stimulates NADH and NADPH oxidase activity in cultured vascular smooth muscle cells. Circ. Res. 1994, 74, 1141-1148.

7. Impellizzeri, D.; Esposito, E.; Mazzon, E.; Paterniti, I.; di Paola, R.; Bramanti, P.; Cuzzocrea, S. Effect of apocynin, a NADPH oxidase inhibitor, on acute lung inflammation. Biochem. Pharmacol. 2011, 81, 636-648.

8. Selemidis, S.; Sobey, C.G.; Wingler, K.; Schmidt, H.H.; Drummond, G.R. NADPH oxidases in the vasculature: Molecular features, roles in disease and pharmacological inhibition. Pharmacol. Ther. 2008, 120, 254-291.

9. Tossi, V.; Cassia, R.; Lamattina, L. Apocynin-induced nitric oxide production confers antioxidant protection in maize leaves. J. Plant Physiol. 2009, 166, 1336-1341.

10. Engels, F.; Renirie, B.F.; Hart, B.A.; Labadie, R.P.; Nijkamp, F.P. Effects of apocynin, a drug isolated from the roots of Picrorhiza kurroa, on arachidonic acid metabolism. FEBS Lett. 1992, $305,254-256$.

11. Valdecir, F.X.; Iguatemy, L.B.; Luiz, M.D.F. Apocynin and related methoxy-catechols as inhibitors of neutrophil-NADPH oxidase in LPS-activated whole blood. Res. J. Biol. Sci. 2007, 2, 360-364.

12. Lu, X.; Wan, S.; Jiang, J.; Jiang, X.; Yang, W.; Yu, P.; Xu, L.; Zhang, Z.; Zhang, G.; Shan, L.; et al. Synthesis and biological evaluations of novel apocynin analogues. Eur. J. Med. Chem. 2011, 46, 2691-2698.

13. Steffen, Y.; Gruber, C.; Schewe, T.; Sies, H. Mono- $O$-methylated flavanols and other flavonoids as inhibitors of endothelial NADPH oxidase. Arch. Biochem. Biophys. 2008, 469, 209-219.

14. Mora-Pale, M.; Weiwer, M.; Yu, J.; Linhardt, R.J.; Dordick, J.S. Inhibition of human vascular NADPH oxidase by apocynin derived oligophenols. Bioorg. Med. Chem. 2009, 17, 5146-5152.

15. Yu, J.; Weiwer, M.; Linhardt, R.J.; Dordick, J.S. The role of the methoxyphenol apocynin, a vascular NADPH oxidase inhibitor, as a chemopreventative agent in the potential treatment of cardiovascular diseases. Curr. Vasc. Pharmacol. 2008, 6, 204-217.

16. Ximenes, V.F.; Kanegae, M.P.; Rissato, S.R.; Galhiane, M.S. The oxidation of apocynin catalyzed by myeloperoxidase: Proposal for NADPH oxidase inhibition. Arch. Biochem. Biophys. 2007, 457, 134-141.

17. Kawahara, T.; Lambeth, J.D. Molecular evolution of Phox-related regulatory subunits for NADPH oxidase enzymes. BMC Evol. Biol. 2007, 7, 178. 
18. Inanami, O.; Johnson, J.L.; Babior, B.M. The leukocyte NADPH oxidase subunit $\mathrm{p} 47^{\text {PHOX}}$ : The role of the cysteine residues. Arch. Biochem. Biophys. 1998, 350, 36-40.

19. Xu, J.; Lu, Y.J.; Shen, W.Z.; Luo, H.B.; Huang, S.C.; Bao, J.L.; Cai, S.H.; Wang, Y.Q. Homology modeling of $\alpha$-glucosidase and its interactions with andrograpolide derivatives. Lett. Drug Des. Discov. 2011, 8, 440-451.

20. Doman, T.N.; McGovern, S.L.; Witherbee, B.J.; Kasten, T.P.; Kurumbail, R.; Stallings, W.C.; Connolly, D.T.; Shoichet, B.K. Molecular docking and high-throughput screening for novel inhibitors of protein tyrosine phosphatase-1B. J. Med. Chem. 2002, 45, 2213-2221.

21. Gehlhaar, D.K.; Verkhivker, G.M.; Rejto, P.A.; Sherman, C.J.; Fogel, D.B.; Fogel, L.J.; Freer, S.T. Molecular recognition of the inhibitor AG-1343 by HIV-1 protease: Conformationally flexible docking by evolutionary programming. Chem. Biol. 1995, 2, 317-324.

22. DesJarlais, R.L.; Seibel, G.L.; Kuntz, I.D.; Furth, P.S.; Alvarez, J.C.; Ortiz de Montellano, P.R.; DeCamp, D.L.; Babe, L.M.; Craik, C.S. Structure-based design of nonpeptide inhibitors specific for the human immunodeficiency virus 1 protease. Proc. Natl. Acad. Sci. USA 1990, 87, 6644-6648.

23. RCSB Protein Data BANK. Available online: http://www.rcsb.org/pdb/explore/explore.do? structureId=1K4U (accessed on 5 September 2011).

24. Verdonk, M.L.; Chessari, G.; Cole, J.C.; Hartshorn, M.J.; Murray, C.W.; Nissink, J.W.; Taylor, R.D.; Taylor, R. Modeling water molecules in protein-ligand docking using GOLD. J. Med. Chem. 2005, 48, 6504-6515.

25. Verdonk, M.L.; Cole, J.C.; Hartshorn, M.J.; Murray, C.W.; Taylor, R.D. Improved protein-ligand docking using GOLD. Proteins 2003, 52, 609-623.

26. Halgren, T.A. Maximally diagonal force constants in dependent angle-bending coordinates. II. Implications for the design of empirical force fields. J. Am. Chem. Soc. 1990, 112, 4710-4723.

27. Clark, M.; Cramer, R.D., III; Opdenbosch, N.V. Validation of the general purpose tripos 5.2 force field. J. Comput. Chem. 1989, 10, 982-1012.

28. Powell, M.J.D. Restart procedures for the conjugate gradient method. Math. Program. 1977, 12, 241-254.

29. Mannhold, R.; Rekker, R.F.; Sonntag, C.; ter Laak, A.M.; Dross, K.; Polymeropoulos, E.E. Comparative evaluation of the predictive power of calculation procedures for molecular lipophilicity. J. Pharm. Sci. 1995, 84, 1410-1419.

30. Ogura, K.; Nobuhisa, I.; Yuzawa, S.; Takeya, R.; Torikai, S.; Saikawa, K.; Sumimoto, H.; Inagaki, F. NMR solution structure of the tandem Src homology 3 domains of $\mathrm{p} 47^{\text {phox }}$ complexed with a p22 phox -derived proline-rich peptide. J. Biol. Chem. 2006, 281, 3660-3668.

31. Groemping, Y.; Lapouge, K.; Smerdon, S.J.; Rittinger, K. Molecular basis of phosphorylation-induced activation of the NADPH oxidase. Cell 2003, 113, 343-355.

32. Bissantz, C.; Folkers, G.; Rognan, D. Protein-based virtual screening of chemical databases. 1. Evaluation of different docking/scoring combinations. J. Med. Chem. 2000, 43, 4759-4767.

(C) 2013 by the authors; licensee MDPI, Basel, Switzerland. This article is an open access article distributed under the terms and conditions of the Creative Commons Attribution license (http://creativecommons.org/licenses/by/3.0/). 